Supplementary Information

\title{
Combined transcriptomic and proteomic analysis of the posterior salivary gland from the southern blue-ringed octopus and the southern sand octopus
}

Brooke Whitelaw ${ }^{1}$, J.M. Strugnell ${ }^{1}$, Pierre Faou ${ }^{2}$, Rute R. da Fonseca ${ }^{4}$, Nathan Hall5,2, Mark Norman ${ }^{5}$, Julian Finn ${ }^{5}$, Ira R. Cooke ${ }^{2,6}$

- Table S1. Orthogroup sequences of the top 20 proteins with the highest abundance isolated from the posterior salivary gland (PSG) of Octopus kaurna. Families include AC (Actin), CP (CAP), HS (Histone), SP (Serine Protease), TR (Tropomyosin) and proteins with no annotated family were classified as UK (Unknown). Family was manually identified through observation of InterPro annotations. pp S3-S4

- Table S2. Orthogroup sequences of the top 20 proteins with the highest abundance isolated from the posterior salivary gland (PSG) of Hapalochlaena maculosa. Families include AC (Actin), CP (CAP), HS (Histone), SP (Serine Protease), TR (Tropomyosin) and proteins with no annotated family were classified as UK (Unknown). Family was manually identified through observation of InterPro annotations. pp S4-S5

- Table S3. Number of sequenced paired-end reads (in millions) for each tissue library of Hapalochlaena maculosa and Octopus kaurna. pp S6

- Fig S1. Maximum likelihood tree of the phylogenetic relationships between homologous CAP putative proteinaceous toxins from Octopus kaurna (orange) and Hapalochlaena maculosa (blue). Bootstrap values $\geq$ 50 shown above nodes. Tree was built with the VT $+\mathrm{G}+\mathrm{I}$ model using amino acid sequences. pp S7

Excel SI files

"Supplementary table 4"

- Table S4. Sequences, which exhibit "toxin-like" features [short $(<150$ amino acids), cysteine rich ( $>3 \%$ cysteines) and secreted (SignalP)] from the posterior salivary gland of Hapalochlaena maculosa and Octopus kaurna. 
"Supplementary tables 5\&6"

- Table S5. Putative toxins isolated from the PSG of Octopus kaurna with their corresponding Trinotate IDs.

- Table S6. Putative toxins isolated from the PSG of Hapalochlaena maculosa with their corresponding Trinotate IDs.

"Supplementary tables 7\&8"

- Table S7. All proteins isolated from Octopus kaurna posterior salivary gland. Includes protein IDs, sequence, cysteine count, length, cysteine \%, indistinguishable proteins, \#proteins, \#peptides, \#unique peptides, sequence coverage, molecular weight, score and iBAQ mean.

- Table S8. All proteins isolated from Hapalochlaena maculosa posterior salivary gland. Includes protein IDs, sequence, cysteine count, length, cysteine \%, indistinguishable proteins, \#proteins, \#peptides, \#unique peptides, sequence coverage, molecular weight, score and iBAQ mean.

"Supplementary table 9\&10"

- Table S9. All prtoein sequences from Octopus kaurna with homology to a transcript isolated in a previous study. Putative toxin IDs are highlighted in yellow.

- Table S10. All protein sequences from Hapalochlaena maculosa with homology to a transcript isolated in a previous study. Putative toxin IDs are highlighted in yellow. 
Table S1: Orthogroup sequences of the top 20 proteins with the highest abundance isolated from the posterior salivary gland (PSG) of Octopus kaurna. Families include AC (Actin), CP (CAP), HS (Histone), SP (Serine Protease), TR (Tropomyosin) and proteins with no annotated family were classified as UK (Unknown). Family was manually identified through observation of InterPro annotations.

\begin{tabular}{llrrrl}
\hline Orthogroup & Trinotate id & Length & \multicolumn{1}{l}{ Cysteine \# } & \multicolumn{1}{l}{ iBAQ } & Family \\
\hline AC20K4 & comp26684_c2_seq3_m.83259 & 375 & 4 & 44609.5 & Actin \\
AC20K1 & comp20288_c2_seq1_m.31146 & 376 & 6 & 140076091.4 & Actin \\
AC20K2 & comp26684_c2_seq4_m.83264 & 375 & 4 & 2777700.571 & Actin \\
AC20K3 & comp26684_c2_seq1_m.83246 & 375 & 4 & 648321.5714 & Actin \\
CP10K1 & comp27308_c0_seq1_m.101997 & 264 & 19 & 54290685.71 & CAP \\
HS20K1 & comp22121_c0_seq1_m.37985 & 121 & 0 & 51131857.14 & Histone \\
HS40K1 & comp24911_c0_seq2_m.55852 & 103 & 0 & 86875714.29 & Histone \\
HS50K1 & comp14212_c0_seq1_m.16916 & 125 & 0 & 174003700 & Histone \\
HY10K1 & comp27648_c0_seq1_m.102959 & 436 & 9 & 897990.8 & Hyaluronidase \\
SP10K1 & comp27296_c0_seq1_m.101938 & 207 & 8 & 68514174.29 & Serine Protease \\
SP20K1 & comp11207_c0_seq1_m.11947 & 255 & 11 & 31414057.14 & Serine Protease \\
SP30K1 & comp20357_c3_seq3_m.31402 & 257 & 11 & 78796428.57 & Serine Protease \\
SP40K1 & comp25408_c0_seq1_m.60376 & 254 & 12 & 73267714.29 & Serine Protease \\
\hline SP50K1 & comp27295_c0_seq1_m.101936 & 254 & 11 & 55028785.71 & Serine Protease \\
SP60K1 & comp27297_c0_seq1_m.101939 & 274 & 11 & 55408457.14 & Serine Protease \\
SP80K1 & comp14350_c1_seq1_m.17313 & 253 & 10 & 74678285.71 & Serine Protease \\
TR10K1 & comp22305_c0_seq2_m.38864 & 284 & 1 & 52355328.57 & Tropomyosin \\
TR20K1 & comp27320_c0_seq1_m.102043 & 174 & 1 & 56695971.43 & Tropomyosin \\
\hline
\end{tabular}




\begin{tabular}{llrrrl}
\hline UK100K1 & comp27294_c0_seq1_m.101933 & 266 & 5 & 889025714.3 & Unknown \\
UK110K1 & comp27298_c0_seq1_m.101943 & 287 & 1 & 12290585.71 & Unknown \\
UK120K1 & comp14353_c0_seq1_m.17320 & 300 & 1 & 116809428.6 & Unknown \\
UK30K1 & comp20288_c1_seq1_m.31142 & 79 & 1 & 110599666.7 & Unknown \\
UK40K1 & comp20288_c1_seq2_m.31143 & 99 & 1 & 113375428.6 & Unknown \\
UK50K1 & comp20288_c1_seq3_m.31145 & 71 & 1 & 163112928.6 & Unknown \\
UK60K1 & comp11211_c3_seq1_m.11958 & 294 & 2 & 18247185.71 & Unknown \\
UK70K1 & comp11247_c1_seq2_m.12024 & 86 & 1 & 10587100 & Unknown \\
UK80K1 & comp24658_c0_seq1_m.53671 & 307 & 2 & 97936800 & Unknown \\
UK90K1 & comp27293_c1_seq1_m.101930 & 322 & 2 & 311743571.4 & Unknown \\
\hline
\end{tabular}

Table S2. Orthogroup sequences of the top 20 proteins with the highest abundance isolated from the posterior salivary gland (PSG) of Hapalochlaena maculosa. Families include AC (Actin), CP (CAP), HS (Histone), SP (Serine Protease), TR (Tropomyosin) and proteins with no annotated family were classified as UK (Unknown). Family was manually identified through observation of InterPro annotations.

\begin{tabular}{llrrrl}
\hline Orthogroup & Trinotate id & Length & Cysteine \# & \multicolumn{1}{l}{ iBAQ } & Family \\
\hline AC1HM1 & comp70911_c2_seq1_m.207412 & 246 & 4 & 127436984 & Actin \\
AC2HM1 & comp69190_c3_seq1_m.168612 & 376 & 6 & 523065000 & Actin \\
CP1HM1 & comp74924_c0_seq1_m.388877 & 273 & 18 & 51893750 & CAP \\
EF1HM1 & comp71908_c0_seq1_m.232960 & 111 & 2 & 1423950000 & EF-hand \\
HS1HM1 & comp70358_c0_seq2_m.195124 & 95 & 1 & 147085000 & Histone \\
HS3HM1 & comp65470_c0_seq4_m.126516 & 125 & 0 & 264161750 & Histone \\
HS4HM1 & comp17737_c0_seq1_m.14626 & 103 & 0 & 132967000 & Histone \\
HS5HM1 & comp20968_c0_seq1_m.20388 & 125 & 0 & 140135200 & Histone \\
HY1HM1 & comp17608_c0_seq1_m.14275 & 432 & 9 & 107868750 & Hyaluronidase \\
\hline
\end{tabular}




\begin{tabular}{llrrrl}
\hline SP2HM1 & comp29613_c0_seq1_m.29982 & 263 & 11 & 149594250 & Serine Protease \\
SP3HM1 & comp48800_c0_seq1_m.60195 & 318 & 18 & 19253900 & Serine Protease \\
\hline SP4HM1 & comp75768_c0_seq1_m.390801 & 262 & 11 & 1488650 & Serine Protease \\
SP5HM1 & comp29651_c0_seq1_m.30075 & 254 & 11 & 122141800 & Serine Protease \\
\hline SP6HM1 & comp29671_c0_seq1_m.30130 & 302 & 18 & 89623175 & Serine Protease \\
SP8HM1 & comp74737_c0_seq1_m.388209 & 259 & 12 & 233434750 & Serine Protease \\
SP8HM2 & comp74766_c0_seq1_m.388320 & 254 & 11 & 18721525 & Serine Protease \\
\hline TR1HM1 & comp66058_c0_seq11_m.132840 & 284 & 1 & 49818750 & Tropomyosin \\
TR2HM1 & comp74829_c0_seq2_m.388572 & 174 & 0 & 106943000 & Tropomyosin \\
UK10HM1 & comp17775_c0_seq1_m.14749 & 281 & 4 & 296415925 & Unknown \\
UK11HM1 & comp17707_c3_seq1_m.14548 & 288 & 1 & 99312750 Unknown \\
UK12HM1 & comp64608_c0_seq1_m.121541 & 317 & 2 & 288257500 & Unknown \\
UK1HM1 & comp35214_c0_seq1_m.40617 & 74 & 5 & 91959500 & Unknown \\
UK2HM1 & comp64558_c0_seq2_m.121377 & 76 & 1 & 91020000 & Unknown \\
UK6HM1 & comp17707_c0_seq1_m.14529 & 285 & 2 & 153052250 & Unknown \\
UK7HM1 & comp74735_c0_seq1_m.388207 & 83 & 0 & 119114700 & Unknown \\
UK9HM1 & comp74972_c0_seq1_m.388993 & 309 & 2 & 94628750 & Unknown \\
\hline
\end{tabular}


Table 3: Number of sequenced paired-end reads (in millions) for each tissue library.

\begin{tabular}{|c|c|c|}
\hline Species & Tissue & $\begin{array}{l}\text { Number of PE Reads } \\
\text { (Million) }\end{array}$ \\
\hline O. kaurna & Salivary gland & 23 \\
\hline O. kaurna & Posterior mantle & 22 \\
\hline H. maculosa & Skin of Dorsal mantle & 24 \\
\hline H. maculosa & Eyeballs & 24 \\
\hline H. maculosa & Gills & 25 \\
\hline H. maculosa & Systemic heart & 24 \\
\hline H. maculosa & Posterior salivary gland & 24 \\
\hline H. maculosa & $\begin{array}{l}\text { Ventral mantle muscle and } \\
\text { skin }\end{array}$ & 27 \\
\hline H. maculosa & Brain & 24 \\
\hline H. maculosa & Anterior salivary glands. & 24 \\
\hline H. maculosa & Branchial hearts & 22 \\
\hline H. maculosa & Renal appendages & 24 \\
\hline H. maculosa & Male reproductive tract & 20 \\
\hline H. maculosa & Digestive gland & 23 \\
\hline
\end{tabular}




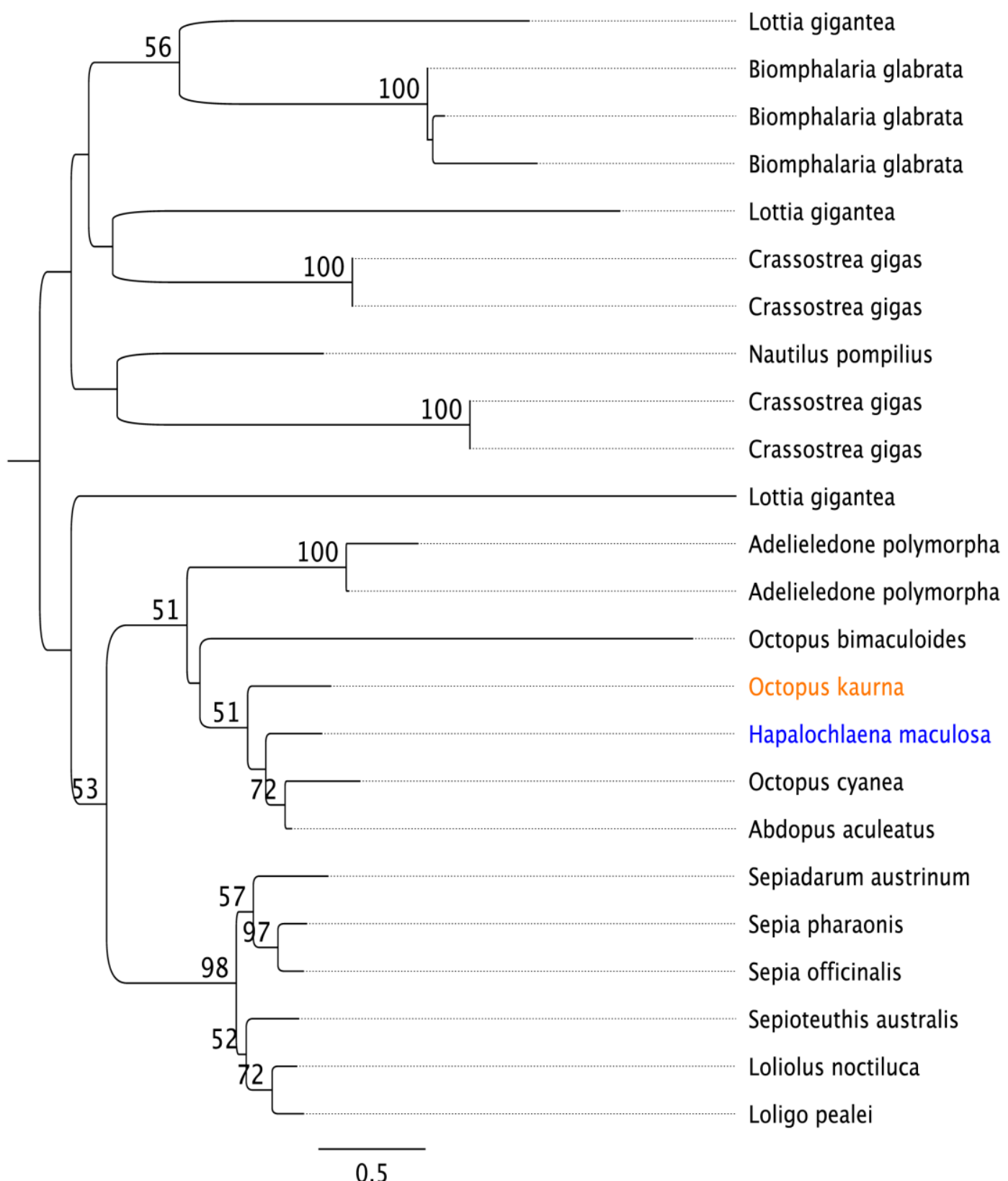

Fig S1. Maximum likelihood tree of the phylogenetic relationships between homologous CAP putative proteinaceous toxins from Octopus kaurna (orange) and Hapalochlaena maculosa (blue). Bootstrap values $\geq 50$ shown above nodes. Tree was built with the $\mathrm{VT}+\mathrm{G}+\mathrm{I}$ model using amino acid sequences. 